\title{
El aprendizaje ubicuo en el proceso de enseñanza aprendizaje
}

\section{Ubiquitous learning in the teaching-learning process}

\author{
Pedro Félix Novoa Castillo ${ }^{1}$ \\ Rosalinn Francisca Cancino Verde ${ }^{2}$ \\ Yrene Cecilia Uribe Hernández ${ }^{3}$ \\ Luzmila Lourdes Garro Aburto 4 \\ Gliria Susana Mendez llizarbe ${ }^{5}$
}

Recibido: 20 de diciembre de 2019. Aceptado: 17 de enero de 2020

\section{RESUMEN}

El presente trabajo busca caracterizar al aprendizaje ubicuo en el proceso de la enseñanza aprendizaje, por ello se propone encuadrarlo en una época contemporánea llena de cambios y pulsiones que permiten no solo su consolidación, sino su particular aprovechamiento. Asimismo, se brindará algunas reflexiones sobre su implicancia dentro de la concepción tradicional del aula, alumno y profesor, asumiendo un cambio paradigmático al relativizar los espacios, los tiempos y los modos de acceso a la información y al escenario de clase. El aprendizaje ubicuo, por tanto, es una especie de modelo que todo docente de estos tiempos debe conocer y practicar en beneficio de los aprendizajes de sus alumnos.

Palabras claves: sistema ubicuo; tecnología ubicua; TIC; aprendizaje ubicuo; enseñanza aprendizaje.

\section{ABSTRACT}

The present work seeks to characterize ubiquitous learning in the teaching-learning process, so it is proposed to frame it in a contemporary era full of changes and drives that allow not only its consolidation, but its particular use. Likewise, there will be some reflections on its implication within the traditional conception of the classroom, student and teacher, assuming a paradigmatic change when relativizing spaces, times and modes of access to information and the class scenario. The ubiquitous learning, therefore, is a kind of model that every teacher of these times must know and practice for the benefit of their students' learning.

Keywords: ubiquitous system; ubiquitous technology; TIC; ubiquitous learning; teaching learning.

1 Universidad Nacional Mayor de San Marcos. Correo electrónico: pnovoac@unmsm.edu.pe, ORCID: https://orcid.org/0000-0003-2186-7458

2 Universidad Nacional Federico Villarreal. Correo electrónico: cancinoverdefrancisca@gmail.com, ORCID: https://orcid.org/0000-0003-02495345

3 Universidad Nacional de Cañete. Correo electrónico: yuribe@undc.edu.pe, ORCID: https://orcid.org/0000-0001-5893-9262

4 Universidad César Vallejo. Correo electrónico: lourdesgarro.ga@gmail.com, ORCID: https://orcid.org/0000-0002-9453-9810

5 Universidad César Vallejo. Correo electrónico: investigadora10@gmail.com, ORCID: https://orcid.org/0000-0001-9919-2003

(c) 2020 Revista Multi-Ensayos. 


\section{INTRODUCCIÓN}

En estos últimos tiempos de acelerado incremento de información y sistemas computacionales, es necesario reflexionar sobre cómo mejoramos y potencializamos el proceso de enseñanza aprendizaje.

El aprendizaje tradicional era memorístico, es decir considerada a los estudiantes como sujetos pasivos. Estudiantes que eran una fuente de receptor de información, y su forma de enseñanza era presencial (aunque actualmente sigue predominando este tipo de enseñanza) en done el estudiante y el docente permanecían en el mismo lugar; este tipo de enseñanza está siendo desplazada por un entorno de aprendizaje ubicuo. Donde el docente pasa a ser un facilitador que puede acompañar a cada estudiante no solo de manera directa sino también de manera indirecta. Es decir, según donde se encuentre mediante los dispositivos que estén accesible en cualquier momento o lugar.

El aprendizaje ubicuo podría considerarse un paradigma educativo que está presente en sociedades a las que no se les restringe el conocimiento, por ello son sociedades informadas, justas y equilibradas puesto que, gozan de las TIC en aulas o ambientes virtuales. Por ello, el aprendizaje ubicuo realizado en sus distintas modalidades - e-learning, m-learning, b-learning y u-learning, es el paradigma educativo que prevalece en las sociedades del conocimiento debido al papel que tienen las TIC como dispositivos pedagógicos, herramientas didácticas y estrategias pedagógicas, pero también por crear escenarios y ambientes virtuales (García, 2015, p.72).

\section{La ubicuidad y los cambios}

Es importante relacionar el aprendizaje ubicuo con los cambios que promoverán nuevos aprendizajes integrándolos al flujo contemporáneo de actividades. Asimismo, reflexionar sobre los cambios que significarán al darse un aprendizaje más situado y contextual, ya que en la enseñanza aprendizaje, la ubicuidad adquirirá una Multi presencia tanto del docente como del estudiante en Multi plataformas. Y a pesar de que los detractores de lo tecnológico vean distorsión y precarización en sus modos de comunicación, la noción de ubicuidad se consolidará en más reflexiva en la medida que los estudiantes tendrán no solo más tiempo para analizar sus actividades, sino que estarán supeditados a sus propios ritmos de aprendizaje. Lo que sí nadie puede negar, incluso los que están en contra de la ubicuidad por un tema de diseminación de información, es que consolidará logros de aprendizajes más colaborativos y un profesor más orientado a los logros de sus estudiantes. Ya que se aprovechará al máximo todo tipo de logro tanto formal, informal, situado y experimental (donde se involucren padres, amigos, familiares, etc.). Incluso propiciarán una interacción con otros profesores del estudiante, transformando así en una suerte de comunidad conectada en torno a un aprendizaje específico (Burbules, 2012).

El aprendizaje tipo ubicuo o también denominado u-learning tiene mucha utilidad para los estudiantes y profesores, ya que permite brindar una enseñanza aprendizaje personalizada en cualquier momento y en cualquier espacio en la medida que se pueda contar con un dispositivo informático como una computadora, una laptop o un dispositivo móvil. Lo contradictorio es que a pesar de tantas bondades 
que implica el aprendizaje ubicuo, este todavía no cuenta con la suficiente cantidad de plataformas para su aprovechamiento máximo (Martínez, Moreno y Miranda, 2010).

Este tipo de aprendizaje ha integrado la tecnología como una estrategia que permitirá a los estudiantes acceder a la información que se desee en cualquier momento y lugar de tal manera que sea el propio estudiante quien asuma la gestión de la información que va acceder. La tecnología ubicua parte de la necesidad de la comunicación social entre las personas y es más rápida dado que permitirá el intercambio de opiniones e ideas entre los estudiantes, debido a su contexto de conectividad tecnológica.

En este escenario, tanto el profesor como los alumnos generan espacios o ambientes, en los cuales, se emplearán TIC y otras herramientas para que la enseñanza aprendizaje fluya de extremo a extremo. Dentro de distintos entornos virtuales donde el ser humano aprende a gestionar. Estos se dan en el momento, a través de medios como el trabajo colaborativo en el intercambio de datos que genera conocimientos gracias a la tecnología, e instrumentos virtuales (TIC) proporcionándole dinamismo.

Se refiere a utilizar la tecnología a favor del estudiante la cual ayudará a un mejor entendimiento y un mejor desenvolvimiento como persona activa en el proceso de enseñanza aprendizaje. Gracias a los grandes cambios que se van dando en torno a la tecnología, sea en cuanto a las herramientas y estrategias pedagógicas novedosas, se ha podido instaurar un nuevo modelo educativo. Este nuevo modelo se basa en un aprendizaje que traspasa las paredes de la institución educativa y llega a darse de manera más significativa en los estudiantes, quienes lograrían sentirse interesados por conocer más y por iniciativa propia; previamente promovidos por un docente correctamente formado y actualizado (García, 2015).

El hecho de capacitarse y adoptar una visión a favor de las tecnologías de la información, así como a esta modalidad de aprendizaje ubicuo, se sustenta en los nuevos y exigentes retos que plantean el espectro económico tanto para alumnos como profesores. Ya que ahora se requiere de más información transformada en conocimiento en menos tiempo. Este conocimiento debe y tiene que ser actualizado, porque el nivel de obsolescencia práctica y teórica es abrumador. Es por ello que la concepción de la enseñanza aprendizaje en estos días implica una constante capacitación tanto del alumno como del profesor en acceso, procesamiento y creación de conocimientos. Se requiere de competencias digitales cada vez más prácticas y específicas, se necesita a la par que los niveles de síntesis y de pensamiento crítico estén acorde a una tecnología que no solo impacta a la educación, sino que la transforma. Esto lanza retos ineludibles para los sistemas de educación busquen no solo actualizarse, sino innovar. Ya que, al preparar a los estudiantes, forjan un destino más promisorio. El u-learning o aprendizaje ubicuo, se considera una suerte de modelo multiforme que, a través del apoyo tecnológico, permite la interacción, acceso y comunicación a través de diversas plataformas. Esto refuerza lo colaborativo y la interacción constante, logrando no solo aprendizajes específicos, sino adquiriendo competencias digitales, tanto individuales como colectivas (Coto, Collazos y Rivera, 2016). 


\section{Características del aprendizaje ubicuo}

Se puede concebir que el aprendizaje ubicuo consiste en un aprendizaje que se lleva a cabo en cualquier lugar y momento. Para ello, se necesita de la tecnología ubicua, es decir todo aquello que abarcan las TIC; ya que mediante estas herramientas se pueden crear espacios virtuales que propicien un aprendizaje donde todos los estudiantes tengan accesibilidad y puedan interaccionar dinámicamente, prescindiendo de un espacio y tiempo determinado. Como sabemos actualmente vivimos inmersos en una época donde abunda el conocimiento, es por esta razón que se necesita que la educación misma, y por ende, los docentes se adapten y acoplen estos recursos a sus enseñanzas, de modo que puedan guiar a los estudiantes tanto en una buena gestión del conocimiento como en el uso apropiado de la tecnología (García, 2015). Entendiéndola como la posibilidad de emplear cualquier tipo de aparato tecnológico en cualquier lugar, en cualquier momento y desde cualquier dispositivo (Alvarez, Unzaga y Durán, 2013).

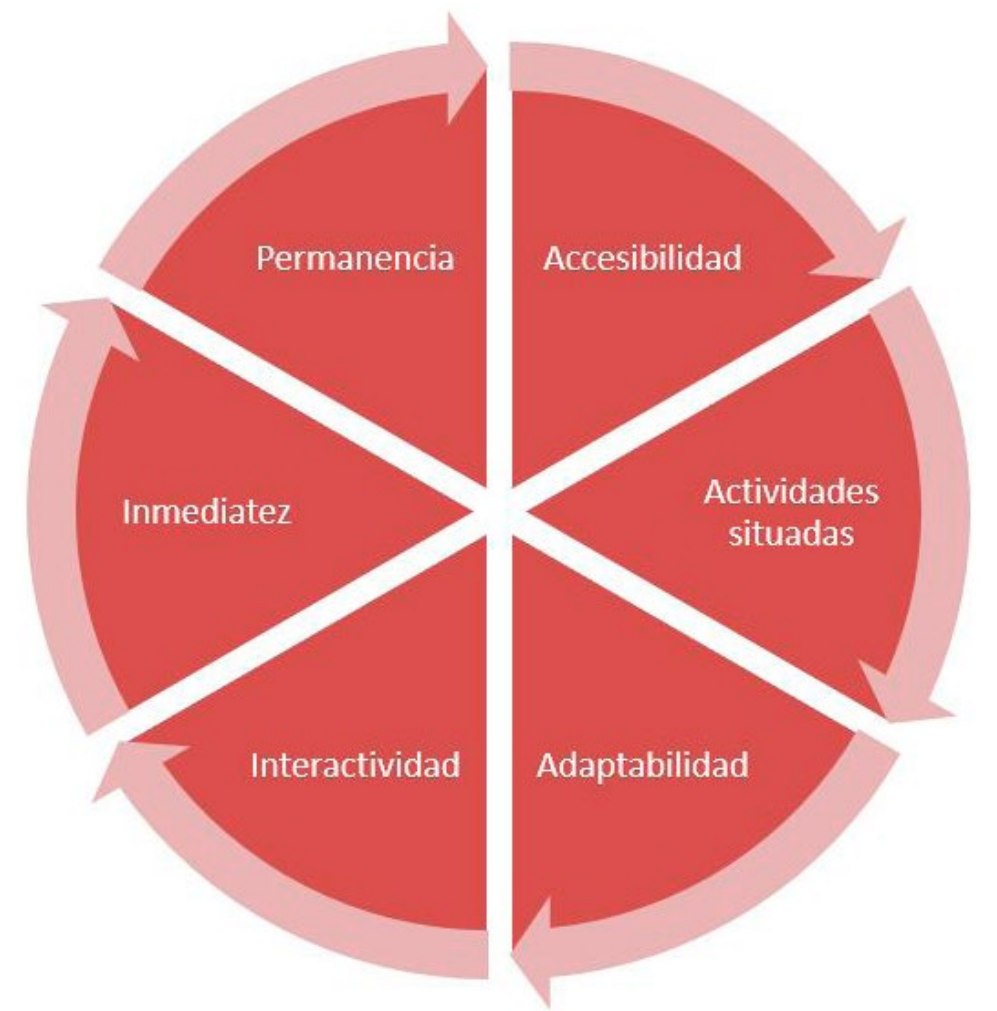

Figura 1. Características del paradigma educativo en el aprendizaje ubicuo

Fuente: Basado de García (2015)

El aprendizaje ubicuo ha tenido aplicaciones en la informática, en la enseñanza de idiomas y en las matemáticas. En esta última ha contribuido a lograr altos niveles de abstracción y razonamiento en los estudiantes (Chicaiza, Escobar y Hernández, 2017). Ya que permite al aprendizaje crecer en cualquier momento y en cualquier lugar, dependiendo del entorno de aplicación y apoyado por herramientas digitales que permiten la inclusión de diferentes actores dentro del proceso de formación. Cabe destacar que el docente debe estas constantemente actualizado con el mundo virtual para una mejora formación significativa del estudiante. 
El aprendizaje ubicuo en su variedad de modalidades, es un paradigma educativo propio de las sociedades postindustriales, pioneras en el rubro de conocimiento e información. Este paradigma adquiere trascendencia gracias al importante papel que han tomado las tecnologías de la información y la comunicación (TIC) en el ámbito educativo, pues han potencializado el aprendizaje a través de sus dispositivos pedagógicos, herramientas y estrategias didácticas (García, 2015). El hecho de aprovechar lo digital no es una postura, es una necesidad, ya que se requiere de más información, más procesamiento y por tanto mayor capacidad o competencias para manejar instrumentos y procedimientos sofisticados (Cadavieco, 2015).

Entre las principales características del aprendizaje ubicuo tenemos (1) la movilidad, ya que los estudiantes pueden conectarse sin necesidad de compartir el mismo lugar, ni estar en un solo lugar, por lo que acceden a la información en cualquier momento y situación; (2) interacción, donde se potencia las acciones interpersonales y la posibilidad de crear comunidades de redes telemáticas tipo virtual de intensa interacción del hombre; (3) colaboración, ya que permite la posibilidad de interrelación con otros participantes en las actividades de aprendizaje; (4) informalidad, constituye aprendizajes que se sujetan a cualquier contexto e inmediatez mediados por la Internet, los dispositivos móviles entre los principales; (5) flexibilidad, ya que no tiene una rigidez en horarios, rutinas ni espacio y contribuye a una personalización del tiempo para el estudio y aprendizaje; (6)los contenidos se pueden llevar, almacenar, enviar, a cualquier plataforma física o virtual (Flores y García, 2014). Sobre todo, en lo relacionado a la distancia, al aprovechar la posibilidad de reducir las brechas de lejanía con lo tecnológico (Hernández, 2016).

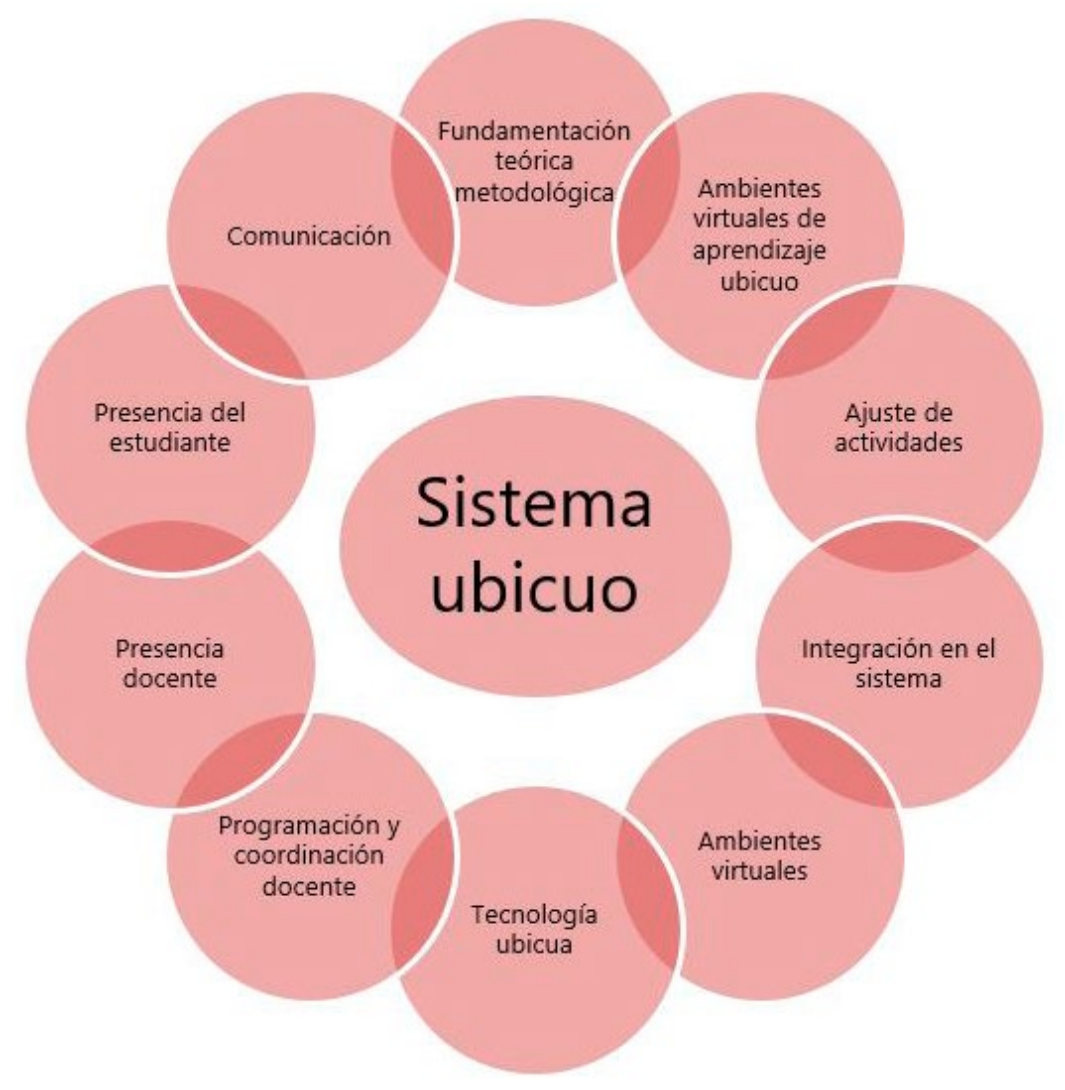

Figura 2. Componentes del sistema ubicuo

Fuente: Basado de Flores y García (2014) 
Adaptarse a lo ubicuo tendrá una relevancia económica, ya que se optimizarán los resultados y logros (Ordóñez, De Clunie y González, 2014). Esto basado en las bondades del conectivismo como una especie de teoría que se beneficia de la teoría del caos, de la complejidad y sobre todo de las redes. Los aprendizajes se dan en constante conexión con otros participantes (Zapata, 2015).

\section{CONCLUSIÓN}

El aprendizaje ubicuo se ha constituido como un modelo de enseñanza aprendizaje para estos tiempos de hiperconexión.

Solo si se adopta la ubicuidad puede la educación estar al ritmo vertiginoso de los cambios no solo tecnológicos, sino sociales y paradigmáticos de la cultura mundial.

El aprendizaje ubicuo tiene múltiples factores que lo hacen pertinente tanto para el docente en el ejercicio de la enseñanza como para el estudiante en el forjamiento de su propio aprendizaje.

La enseñanza aprendizaje desde la ubicuidad permite una mayor flexibilidad, mayor rapidez y mejor personalización de los aprendizajes.

\section{REFERENCIAS}

Alvarez, M. M., Unzaga, S., \& Durán, E. B. (2013). Un enfoque basado en modelos para representar el contexto en aplicaciones de aprendizaje ubicuo. Proceedings of IX Jornadas de Ciencias $y$ Tecnologías de Facultades de Ingeniería del NOA, 97-105.

Burbules, N. C. (2012). El aprendizaje ubicuo y el futuro de la enseñanza. Encounters on education, 13.

Cadavieco, J. F. (2015). Modelos de investigación en contextos ubicuos y móviles en Educación Superior. Educatio Siglo XXI, 33(2 Julio), 329-332.

Chicaiza, R. P. M., Escobar, M. C. C., \& Hernández, W. G. (2017). Espacio virtual iconográfico de aprendizaje ubicuo orientado al desarrollo del pensamiento lógico en bachillerato general unificado. REFCalE: Revista Electrónica Formación y Calidad Educativa. ISSN 1390-9010, 5(2), 85-98.

Coto, M., Collazos, C. A., \& Rivera, S. M. (2016). Modelo Colaborativo y Ubicuo para apoyar los procesos de enseñanza-aprendizaje a nivel Iberoamericano. Revista de Educación a Distancia, (48).

Flores Ortiz, Á., \& García Martínezi, A. (2017). Sistema de aprendizaje ubicuo en ambientes virtuales. Revista Cubana de Educación Superior, 36(2), 27-40.

García, D. (2015). Tecnología y aprendizaje ubicuo. Revista Iberoamericana de Sistemas, Cibernética e Informática, 12(1), 66-73. Recuperado de http://www.iiisci.org/journal/CV\$/risci/pdfs/CA151ED15. pdf

Hernández, W. G. (2016). Análisis de los entornos virtuales de enseñanza-aprendizaje a partir del enfoque histórico cultural. Campus Virtuales, 5(2), 44-57.

Martínez, H. A. V., Moreno, F. J. T., \& Miranda, C. A. L. (2010). Aprendizaje ubicuo en la enseñanza de las matematicas. Revista Estudios Culturales, (5), 123-136. 
Ordóñez, C. A. C., De Clunie, G., \& González, C. S. (2014). Red Iberoamericana de apoyo a los procesos de enseñanza-aprendizaje de competencias profesionales a través de entornos Ubicuos y Colaborativos. Campus Virtuales, 3(2), 108-116.

Zapata-Ros, M. (2015). Teorías y modelos sobre el aprendizaje en entornos conectados y ubicuos. Bases para un nuevo modelo teórico a partir de una visión crítica del "conectivismo". Education in the Knowledge Society, 16(1), 69-102. 